Approved for public release; distribution is unlimited.

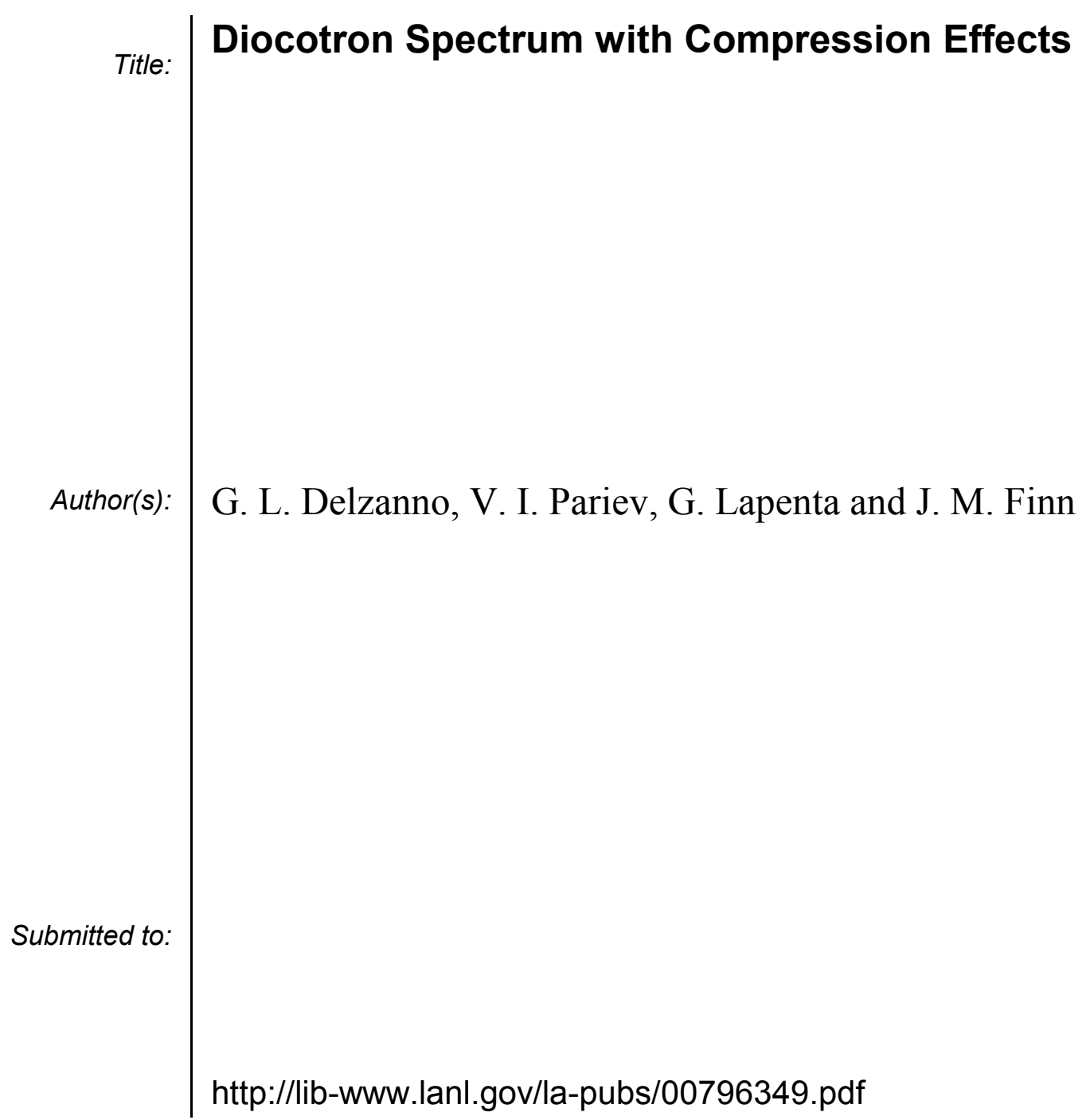

Los Alamos National Laboratory, an affirmative action/equal opportunity employer, is operated by the University of California for the U.S. Department of Energy under contract W-7405-ENG-36. By acceptance of this article, the publisher recognizes that the U.S. Government retains a nonexclusive, royaltyfree license to publish or reproduce the published form of this contribution, or to allow others to do so, for U.S. Government purposes. Los Alamos National Laboratory requests that the publisher identify this article as work performed under the auspices of the U.S. Department of Energy. Los Alamos National Laboratory strongly supports academic freedom and a researcher's right to publish; as an institution, however, the Laboratory does not endorse the viewpoint of a publication or guarantee its technical correctness. 


\title{
Diocotron Spectrum with Compression Effects
}

\author{
G. L. Delzanno*, V. I. Pariev ${ }^{\dagger}$, G. Lapenta** and J. M. Finn ${ }^{\ddagger}$ \\ ${ }^{*}$ Istituto Nazionale di Fisica per la Materia (INFM) and Dipartimento di Energetica, Politecnico di \\ Torino, Corso Duca degli Abruzzi 24, 10129 Torino, Italy \\ ${ }^{\dagger}$ Theoretical Division, Los Alamos National Laboratory, Los Alamos, NM 87545, USA and \\ Lebedev Physical Institute, Leninsky Prospect 53, Moscow B-333, 117924, Russia \\ ${ }^{* *}$ Istituto Nazionale di Fisica per la Materia (INFM) and Theoretical Division, Los Alamos \\ National Laboratory, Los Alamos, NM 87545, USA \\ ${ }^{\ddagger}$ Theoretical Division, Los Alamos National Laboratory, Los Alamos, NM 87545, USA
}

\begin{abstract}
The diocotron spectrum for a simplified model of Malmberg-Penning traps that includes compression effects due to end curvature is investigated herein. Performing an initial value treatment, we find that there is a class of length profiles for which the linearized eigenvalue equation of the model can be integrated in quadratures (integrable case). In this case, there is only algebraic growth when the effective angular frequency has a maximum (hollow profile) or a minimum, and the model is mathematically equivalent to the zero curvature (2D Euler) case. Furthermore, we study profiles that are slightly different from the integrable one (the difference being characterized by a small parameter, $\varepsilon$ ), finding that the frequency of the unstable $l=1$ mode scales as $\varepsilon^{2 / 3}$. Analytical calculations and numerical simulations are found in remarkable agreement.
\end{abstract}

\section{INTRODUCTION}

The analogy between the 2D Euler equations for an incompressible and inviscid fluid and the classical equations for the evolution of a non-neutral plasma (2D drift-Poisson model) in a Penning trap is well known [1]. According to the linear theory, the 2D driftPoisson model has demonstrated to be satisfactory only for (exponentially unstable) perturbations with azimuthal mode number $l>1$. On the other hand, it can be proved theoretically that perturbations with mode number $l=1$ are always stable, regardless of the initial equilibrium density profile [1]. Experiments, however, show that the linear growth of the $l=1$ mode is exponential [2].

New fluid-dynamics models have been developed for a non-neutral plasma, trying to solve the problem of the $l=1$ diocotron instability $[3,4]$. These works show that a possible explanation of the instability comes from the finite curvature of the ends of the plasma column due to the confining voltage (i.e. compression effects).

The present study investigates the modification of the low-frequency branch of the diocotron spectrum when these compression and finite electron temperature effects are taken into account. 


\section{PHYSICAL MODEL}

We focus our attention on a simplified version of the model developed by some of the authors in Ref. [4]. In normalized units, the model is the following:

$$
\left\{\begin{array}{l}
\frac{\partial \sigma}{\partial t}=-\mathbf{V}_{\perp} \cdot \nabla_{\perp} \sigma \\
\mathbf{V}_{\perp}=\widehat{\mathbf{e}}_{z} \times \nabla_{\perp} \phi_{\mathrm{eff}} \\
\nabla_{\perp}^{2} \phi=\frac{\sigma}{\mathcal{L}_{0}} \\
\phi_{\mathrm{eff}}=\phi+\alpha \log \frac{\mathcal{L}_{0}}{\mathcal{L}_{0, r=0}}
\end{array}\right.
$$

For a detailed derivation of this model we refer to Refs. [4, 5, 6]. Compression effects are retained in the terms depending on the normalized temperature $\alpha$ (assumed to be uniform) and on the normalized effective plasma length $\mathcal{L}_{0}(r)$. The relation between physical and dimensionless quantities can be found in Ref. [5]. The effective plasma length is assumed fixed in time and this makes the model 2D.

The motivation of this study is that this approximated model is still able to capture the features of the $l=1$ diocotron instability (as shown in Ref. [4]) but, since it is 2D, it is possible to perform analytical calculations to match numerical simulations. Our goal is to get some insights on the effects of both temperature and length profile on the diocotron spectrum that will help us in the study of the complete model of Ref. [4].

\section{ALGEBRAIC INSTABILITY IN INTEGRABLE CASE}

First, we perform an analysis similar to the one by Smith and Rosenbluth [7]. Applying the Laplace transformation to the linearized time-evolution equation that can be deduced from model (1), one obtains:

$$
(\omega-l \Omega)\left[\frac{1}{r} \frac{d}{d r}\left(r \frac{d \delta \phi_{\omega}}{d r}\right)-\frac{l^{2}}{r^{2}} \delta \phi_{\omega}\right]+\frac{l}{r} \frac{\sigma_{0}^{\prime}}{\mathcal{L}_{0}} \delta \phi_{\omega}=i \frac{\delta \sigma(r, 0)}{\mathcal{L}_{0}}
$$

where the prime means derivative with respect to $r$ and $\delta \phi_{\omega}$ and $\delta \sigma(r, 0)$ are respectively the Laplace transformed perturbation of the potential and the initial perturbation of the line integrated charge density. A new effective angular frequency has been defined

$$
\Omega(r)=\omega_{E}(r)+\frac{\alpha}{r} \frac{\mathcal{L}_{0}^{\prime}(r)}{\mathcal{L}_{0}(r)}
$$

where $\omega_{E}(r)=\phi_{c 0}^{\prime}(r) / r$ is the $\mathbf{E} \times \mathbf{B}$ equilibrium angular frequency in the central plane of the trap. In the limit of $\mathcal{L}_{0}=$ const, Eq. (2) reduces to the classical Euler case.

After some algebra, it can be shown that under a certain condition Eq. (2) can be integrated in quadratures, i.e. $\delta \phi_{\omega}(r)=r[\omega-\Omega(r)]$ is a solution of the problem for $l=1$. We refer to this case as the integrable case. The condition is that the following equation be satisfied: 


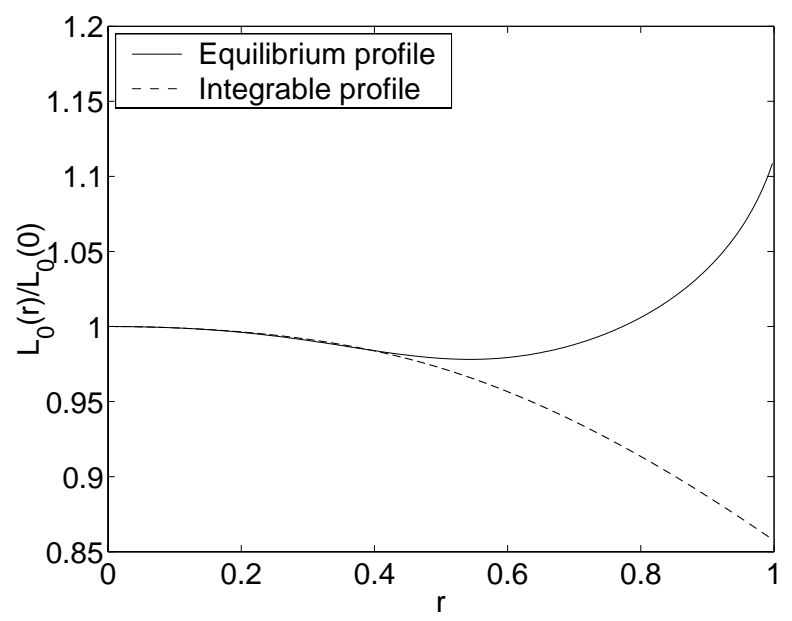

FIGURE 1. Effective length profiles.

$$
\chi^{\prime \prime}+\frac{\chi^{\prime}}{r}-\frac{\chi}{r^{2}}=\frac{\sigma_{0}}{\mathcal{L}_{0}} \frac{\chi}{\alpha},
$$

where $\chi=\mathcal{L}_{0}^{\prime} / \mathcal{L}_{0}$. In this case, provided that the effective angular frequency has a stationary point, the system is algebraically unstable and the mode perturbation grows asymptotically proportionally to $\sqrt{t}$ (as for the classical 2D Euler case [7]). This result has been confirmed numerically.

Given the equilibrium initial parameters, Eq. (4) can be used to obtain the integrable length profile and in general this profile will be different from the one that comes from the equilibrium solution for a given configuration of the Penning trap. In order to make these two profiles as close as possible inside the plasma radius (which is the most important region, as pointed out in Ref. [3]) we use the boundary conditions $\chi(0)=0$ and $\chi^{\prime}(0)=\mathcal{L}_{0, \text { eq }}^{\prime \prime}(0) / \mathcal{L}_{0, \text { eq }}(0)$. Note however that one could in principle choose the geometry of the trap (which determines the equilibrium profile) to match the integrable length profile $\mathcal{L}_{0, \text { integ }}(r)$.

Figure 1 shows the length profiles. The equilibrum length profile is calculated for the same geometrical and physical parameters of Ref. [4], with the choice of confining voltage $V=50 \mathrm{~V}$ and temperature $\Theta=7.5 \mathrm{~V}$. Accordingly, we choose the normalized temperature $\alpha=0.42$ and the normalized equilibrum density profile [3, 4]:

$$
n_{0}(r)=\frac{\sigma_{0}}{\mathcal{L}_{0}}=\left\{\begin{array}{l}
n_{0}(0)\left[1-\left(\frac{r}{r_{p}}\right)^{2}\right]^{2}\left[1+(\mu+2)\left(\frac{r}{r_{p}}\right)^{2}\right], \\
0, \quad r \leq r_{p} \\
r>r_{p},
\end{array}\right.
$$

with $n_{0}(0)=6.2, \mu=3$ and $r_{p}=0.59$. 


\section{INITIAL VALUE TREATMENT}

In Eq. (2), the Laplace transformation of the perturbation of the potential is defined as $\delta \phi_{\omega}(r)=\int_{0}^{+\infty} e^{i \omega t} \delta \phi(r, t) d t$, while the inverse Laplace transformation is given by $\delta \phi(r, t)=\int_{-\infty+i \eta}^{+\infty+i \eta} \delta \phi_{\omega}(r) e^{-i \omega t} \frac{d \omega}{2 \pi}$ where $\eta$ is a large enough real positive number, such that all irregular points of the function $\delta \phi_{\omega}$ are located below the line $\operatorname{Im} \omega=\eta$ in the complex plane of $\omega$.

We introduce the function $\psi(r, \omega)=r \delta \phi_{\omega}(r)$ and solve the second order equation for $\psi$ induced by Eq. (2). Unlike $\delta \phi_{\omega}$, the solutions for $\psi$ are bounded at $r \longrightarrow 0$, which is necessary for Eq. (6) below.

We perform our analysis in the vicinity of the stationary point $r_{s}\left(\omega_{s}=\Omega\left(r_{s}\right)\right)$, by using the method of matched asymptotic expansions for $\psi(r, \omega)$. For simplicity, the initial perturbation is chosen to be localized, $\delta \sigma(r, 0)=\sigma_{i} \delta\left(r-r_{i}\right)$, as it is done in Ref. [8]. This method gives the following result

$$
\begin{aligned}
& \delta \phi(r, t)=-\frac{i r_{s} \sigma_{i}}{3 r \mathcal{L}_{0}\left(r_{i}\right)} \int_{C} \frac{e^{-i \omega t}}{\omega-\Omega\left(r_{i}\right)} \times \\
& \left(\psi_{r}(r, \omega) \psi_{s}\left(R_{w}, \omega\right)-\frac{3 \pi}{2} i\left(\frac{\Omega^{\prime \prime} \mid r_{s}}{2 \Delta}\right)^{3 / 2} \psi_{r}\left(R_{w}, \omega\right) \psi_{r}(r, \omega)-\psi_{r}\left(R_{w}, \omega\right) \psi_{s}(r, \omega)\right) \times \\
& \frac{\psi_{r}\left(r_{i}, \omega\right) \psi_{s}(0, \omega)-\psi_{s}\left(r_{i}, \omega\right) \psi_{r}(0, \omega)}{\psi_{r}(0, \omega)\left(-\frac{3 \pi}{2} i\left(\frac{\left.\Omega^{\prime \prime}\right|_{s}}{2 \Delta}\right)^{3 / 2} \psi_{r}\left(R_{w}, \omega\right)+\psi_{s}\left(R_{w}, \omega\right)\right)-\psi_{s}(0, \omega) \psi_{r}\left(R_{w}, \omega\right)} \frac{d \omega}{2 \pi} .
\end{aligned}
$$

Here the contour $C$ goes around the vertical branch cut attached to the point $\omega=\omega_{s}$ in the complex plane of $\omega . \psi_{r}(r, \omega)$ and $\psi_{s}(r, \omega)$ are two linear independent solutions of the homogeneous part of the Laplace transformed equation such that $\psi_{r} \sim O\left(\left(r-r_{s}\right)^{2}\right)$ and $\psi_{s} \sim O\left(1 /\left(r-r_{s}\right)\right)$ in the vicinity of $r_{s}$. We have also introduced $\Delta=\omega-\omega_{s}$ and $\left.\Omega^{\prime \prime}\right|_{r_{s}}=\left.\frac{d^{2} \Omega}{d r^{2}}\right|_{r=r_{s}}$.

Two merging singular points are present when $r$ approaches $r_{s}$ and the contour of integration in the complex plane of $r$ should always go between these two points [9].

We expand in power of $\Delta$ the integrand of Eq. (6) and perform the integral. Each term of the expansion contributes as $\int_{C} \Delta^{v} e^{-i \omega t} \frac{d \omega}{2 \pi} \propto t^{-v-1} e^{-i \omega_{s} t}$. The algebraic instability $(v<-1)$ is only possible if the term proportional to $\Delta^{-3 / 2}$ in the denominator of expression (6) vanishes, i.e. if $\psi_{r}(0, \omega)=0$ or $\psi_{r}\left(R_{w}, \omega\right)=0$. In the integrable case $\psi_{r}=r^{2}\left(\Omega(r)-\omega_{s}\right)$ and, therefore, $\psi_{r}(0, \omega)=0$. Thus, in the integrable case, the leading term of the expansion has $v=-3 / 2$ (from the numerator of Eq. (6)) and the calculation of the contour integral leads to growth $\propto \sqrt{t}$. Exact calculations of the contour integral (6) recover the asymptotic formula obtained by the stationary phase method in Ref. [7].

When the length profile deviates only a little from the integrable profile (we refer to this case as non-integrable case), $\mathcal{L}_{0}=(1-\varepsilon) \mathcal{L}_{0, \text { integ }}+\varepsilon \mathcal{L}_{0, \text { eq }}$, where $\varepsilon$ is a small parameter, the perturbation to the solutions of Eq. (2) are $\propto \varepsilon$. In particular, $\delta \psi_{r} \propto \varepsilon$ and $\psi_{r}(0, \omega) \propto \varepsilon$. Since $\psi_{r}(0, \omega) \neq 0$, algebraic growth does not occur in the non-integrable 


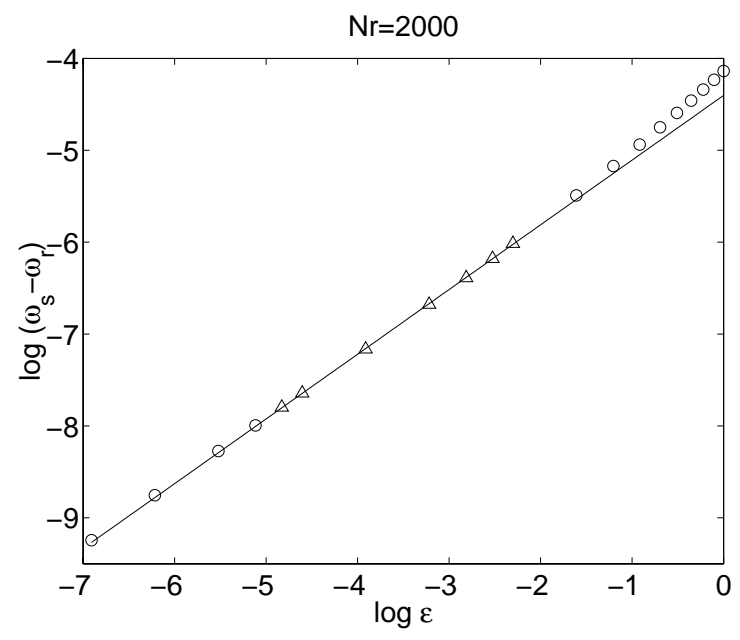

FIGURE 2. Frequency shift of the unstable $l=1$ mode, log-log scale.

case (continuum modes near the endpoint $\omega=\omega_{s}$ phase mix according to $t^{-1 / 2}$ in this case). The zeroes of the denominator in Eq. (6) determine the discrete eigenfrequencies. One can see that for small $\varepsilon$ the eigenfrequencies exist with $\Delta \propto \varepsilon^{2 / 3}$ (as predicted by Smith in Ref. [10] for an ad-hoc model and seen in Ref. [3]). These eigenfrequencies are located close to the edge of the continuum $\omega_{s}$.

Numerical simulations have been done to compare with analytical predictions. Specifically, Fig. 2 shows the frequency shift of the real frequency $\left(\omega_{r}\right)$ of the unstable $l=1$ mode with respect to the edge of the continuum, for $\varepsilon$ between 0 and 1 . We perform the best fit with the least squares method only for the points marked with triangles, since for very small $\varepsilon$ there is a loss of accuracy and for large $\varepsilon$ the perturbation theory fails. We find $\omega_{s}-\omega_{r} \propto \varepsilon^{0.70}$, in good agreement with the result $\omega_{s}-\omega_{r} \propto \varepsilon^{2 / 3}$ obtained from Eq. (6).

Figure 3 shows the growth rate $(\gamma)$ of the unstable $l=1$ mode as a function of $\varepsilon$. Remarkably, we find $\gamma \propto \varepsilon^{0.65}$, in excellent agreement with $\gamma \propto \varepsilon^{2 / 3}$. One can also notice that the integrable case is a very specific case and as soon as one moves a little bit from this profile, an exponential growth is found.

Figure 4 shows the diocotron spectrum for $\varepsilon=1$, corresponding to $\mathcal{L}_{0}=\mathcal{L}_{0 \text {,eq }}$. The upper edge of the continuum splits in two complex conjugate discrete modes (with real part of the frequency that decreases as $\varepsilon$ increases from 0 to 1). It is seen in Fig. 4 that another discrete mode appears at the lower edge of the continuum (in the integrable case this mode is the stable diocotron mode). As predicted analytically and confirmed numerically, its real frequency shift (with respect to the lower edge of the continuum) scales proportionally to $\varepsilon$.

\section{REFERENCES}

1. Davidson, R. C., An Introduction to the Physics of Nonneutral Plasmas, Addison-Wesley, Redwood City, 1990. 


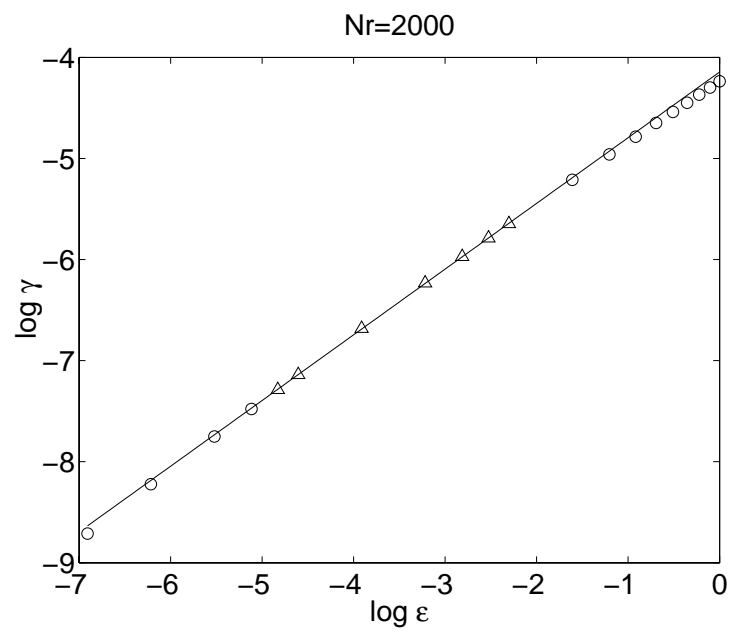

FIGURE 3. Growth rate of the unstable $l=1$ mode, log-log scale.

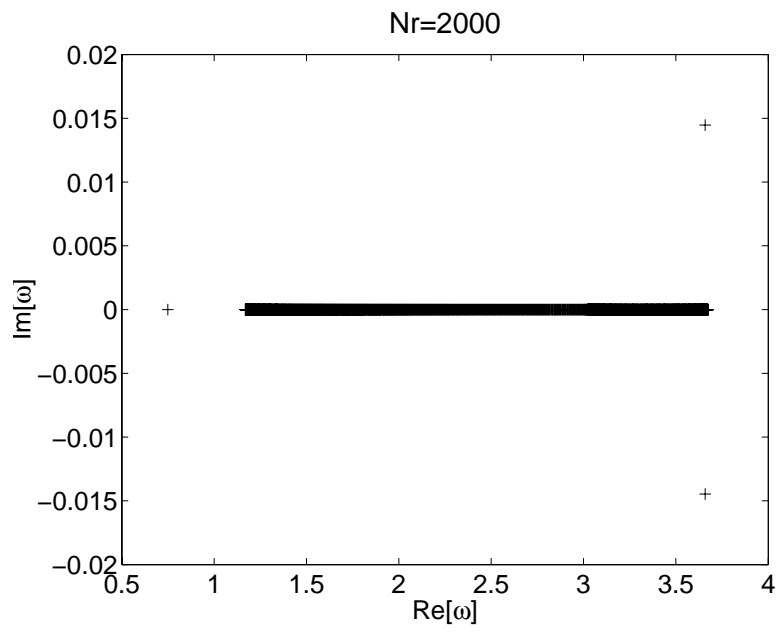

FIGURE 4. Spectrum.

2. Driscoll, C. F., and Fine, K. S., Phys. Fluids B 2, 1359-1366 (1990).

3. Finn, J. M., del-Castillo-Negrete, D., and Barnes, D. C., Phys. Plasmas 6, 3744-3758 (1999); Finn, J. M., del-Castillo-Negrete, D., and Barnes, D. C., Phys. Rev. Lett. 84, 2401-2404 (2000).

4. Coppa, G. G. M., D’Angola, A., Delzanno, G. L., and Lapenta, G., Phys. Plasmas 8, 1133-1141 (2001).

5. Lapenta, G., Delzanno, G. L., and Finn, J. M., "Non-linear PIC Simulation in a Penning Trap", these proceedings.

6. Coppa, G. G. M., D’Angola, A., Delzanno, G. L., and Lapenta, G., "Rigorous Fluid Model for 3D Analysis of the Diocotron Instability", these proceedings.

7. Smith, R. A., and Rosenbluth, M. N., Phys. Rev. Lett. 64, 649-652 (1990).

8. Case, K. M., Phys. Fluids 3, 143-148 (1960).

9. Istomin, Ya. N., and Pariev, V. I., Mon. Not. Roy. Astron. Soc. 281, 1-26 (1996).

10. Smith, R. A., Phys. Fluids B 4, 287-289 (1992). 